\title{
The Certain Possibility of Compensation of the Frequency Response of a LP S-K Biquad Based on the Signal Flow Graph
}

\author{
Bohumil Brtnik, David Matousek \\ Department of Electrical Engineering \\ University of Pardubice \\ 53002 Pardubice Czech Republic \\ bohumil.brtnik@upce.cz,david.matousek@upce.cz
}

\begin{abstract}
The Sallen-Key low-pass filter structures of the even orders have one general problem. This problem is a decreasing of the attenuation at high frequencies. This denoted decreasing of the attenuation is typical for the filters of the even orders only. Therefore, this ineligible frequency response is typical for the filters assembled exclusively from biquads.

The reasons for this effect are explained in this paper and then verified by simulations. The Sallen-Key filter with fully differential $\mathrm{i} / \mathrm{o}$ amplifier is a structure that eliminates decreasing of the attenuation at high frequencies. Firstly, this circuit solution is used as a reference circuit. Secondary, a structure that does not have this property is designed based on the signal flow graph. The resulted frequency response of this proposed structure is verified by computer simulation. These simulations were performed by MicroCap version 11.
\end{abstract}

Keywords-low-pass Sallen-Key filter structure; real operational amplifier; frequency response; fully differential i/o amplifier

\section{INTRODUCTION}

The low-pass Sallen-Key filter structures [1], [2], [3] based on a polynomial from biquads have different circuit solution for the odd order and even order variant. The low-pass filters of the even orders are constructed from biquads only. But low-pass filters of the odd orders contain not only biquads but also passive low pass filter ( $\mathrm{RC}$ network) at the input.

The absence of these RC networks has a key effect to the frequency response of the low-pass filters. The low-pass filters of the even orders produce decreasing of the attenuation at high frequencies. But the low-pass filters of the odd orders don't decrease their attenuation at high frequencies. These situations are documented in Fig. 1 and Fig. 2.

Fig. 1 illustrates an example of the magnitude plot of the low-pass filters of the 1 st and the 3rd order (generally, the low-pass filters of the odd orders) [4], [5], [6].

Fig. 2 illustrates an example of the magnitude plot of the low-pass filters of the 2 nd and the 4 th order (generally, the low-pass filters of the even orders).

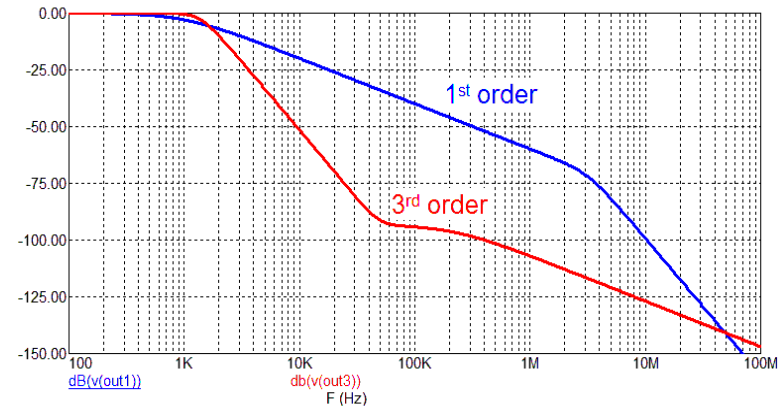

Fig. 1. Magnitude plot of the low-pass filters of the odd orders.

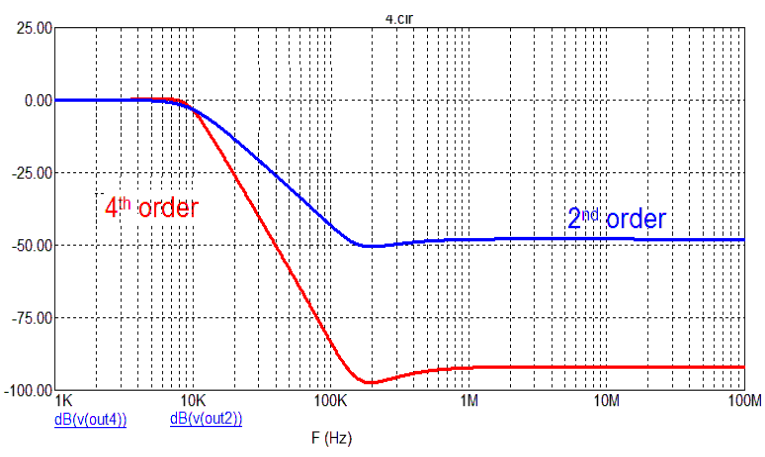

Fig. 2. Magnitude plot of the low-pass filters of the even orders.

At the high frequencies, all capacitors behave like a short circuit and the operational amplifier (OPA) loses the open loop gain $A$. We consider the equivalent circuits in individual frequency ranges, especially the equivalent circuit in the frequency range around the transient frequency [7]. These equivalent circuits accord to Fig. 3.

Fig. $3 \mathrm{~b}$ is a schematic diagram of a biquad $(G, C$ and $2 \cdot C$ are values of the conductance and capacitance of used resistors and capacitors). Fig. $3 \mathrm{c}$ is a simplified schematic diagram of the biquad at high frequencies (all capacitors are substituted to a short circuit). Fig. $3 d$ is an equivalent circuit of the biquad at high frequencies (operational amplifier is modelled as a voltage 
controlled voltage source with the amplification factor $A$ and series conductance $g$ ). Fig. $3 \mathrm{e}$ is an equivalent circuit of the biquad and its admittance matrix at the highest frequencies (operational amplifier loses its amplification factor, now).

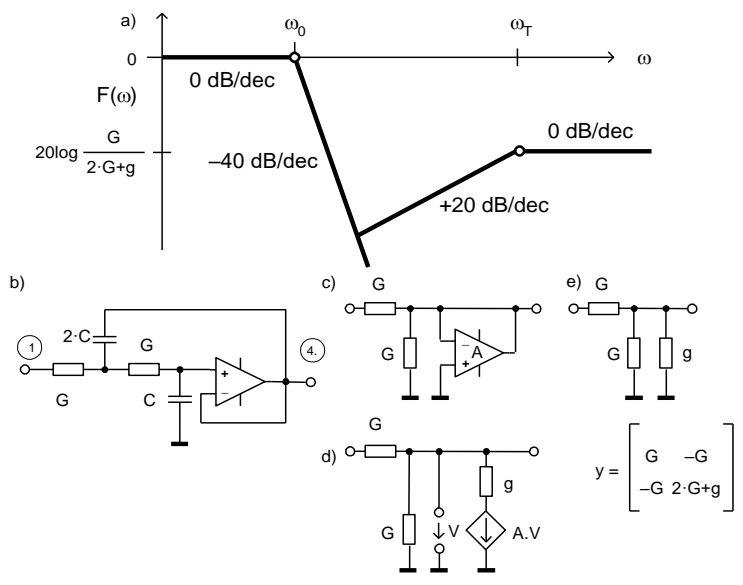

Fig. 3. Frequency response of the second order filter (biquad) and its equivalent circuits in individual frequency ranges.

Therefore, the filter gradually goes from "normal mode" to the frequency-independent voltage divider. This situation is shown in Fig. 3a. At the highest frequencies, the input and output nodes are connected directly by conductance $G$ and the output node is connected to ground by a parallel combination of conductance $G$ and $g$. Therefore, the magnitude of the voltage transfer ratio at the highest frequencies is:

$$
\begin{gathered}
F_{\text {inf }}(s)=20 \cdot \log \left|\lim _{\omega \rightarrow \infty} \frac{V_{\text {OUT }}(s)}{V_{I N}(s)}\right|=20 \cdot \log \left|\frac{-(-G)}{2 \cdot G+g}\right|= \\
=20 \cdot \log \frac{G}{2 \cdot G+g}
\end{gathered}
$$

where: $F_{\text {inf }}$ is the magnitude of the voltage transfer ratio close to infinity frequency, $V_{I N}, V_{O U T}$ are the input and output voltages, $g$ is the series output conductance of the operational amplifier, $G$ is the conductance of a working resistor in the filter.

\section{Proposed CIRCUIT DiAgRAM}

The signal flow graph [3], [8], [9], [10] of the proposed biquad on Fig. 4a shows, that the signal passes from input to output independently to a value of the gain $A$. Therefore, the signal passes from input to output even if the gain $A$ has value zero (the symbol $A$ represents the open loop gain of the operational amplifier). This fact leads to a reduction of the attenuation of the filter.

Now, if two operational amplifiers are used then the signal flow graph is changed to Fig. 4b. The signal flow graph in Fig. 4 b shows that the output of the biquad (in node 5) is fully separated from the output to the feedback branches (in node 4). Whereas if the amplification factor $A$ has value zero, then the input signal can go to the output through the branch with transfer $s 2 \cdot C$. But the operational amplifier prevents the propagation of a signal via the branch with the transfer $A$, because the amplifier amplifies only in one direction while capacitor transmits signals in both directions.

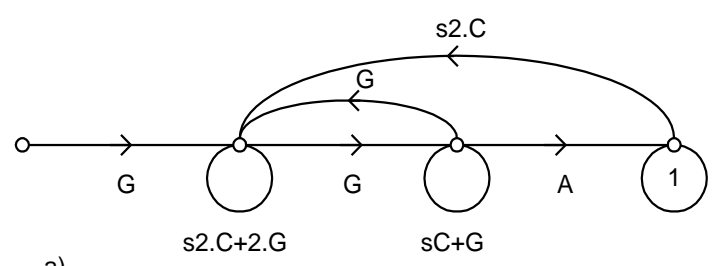

a)

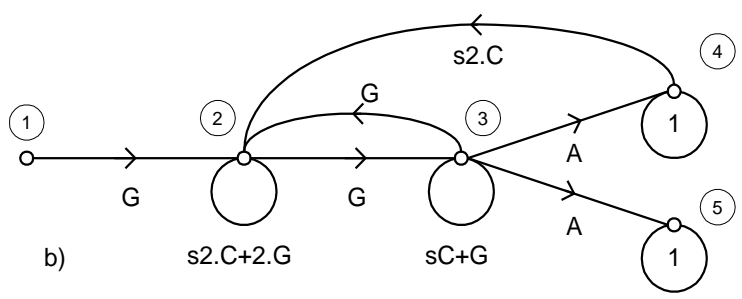

Fig. 4. Signal flow graph of the biquad correspond to a) Fig. 3 and b) Fig. 5.

The pair of the operational DISO (Dual-Input SingleOutput) amplifiers connected as two voltage followers can be connected in parallel at the input as shown in Fig. 5.

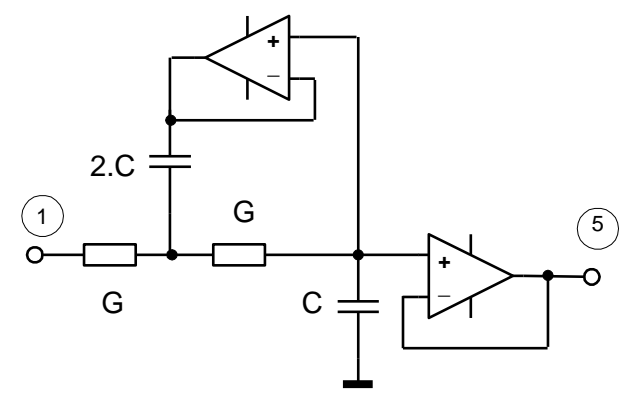

Fig. 5. Proposed circuit with a pair of DISOs as voltage followers.

Simulation by spice-like program MicroCap version 11 verified the proposed solution. Convectional DISO devices LF155 (Gain Bandwidth Product is $G B P=2.5 \mathrm{MHz}$ ) were used for these simulations. Fig. 6 represents a schematic diagram of the low-pass filter with cutoff frequency $f_{C}=100 \mathrm{kHz}$.

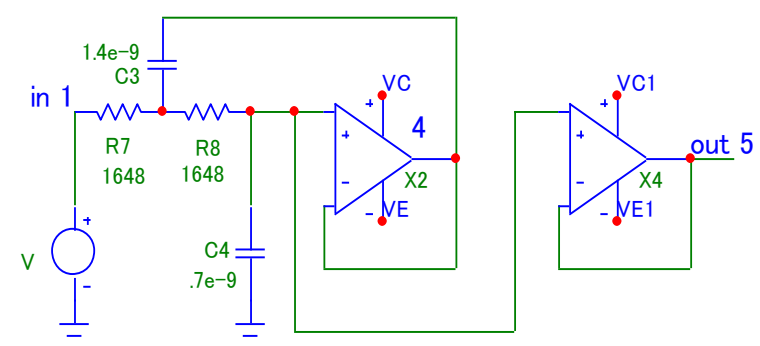

Fig. 6. Schematic diagram of the circuit for simulation of the proposed lowpass Sallen-Key biquad with a pair of the voltage DISO followers. 
The decreasing in the attenuation at high frequencies did not occur for output according to the node 5 (see Fig. 7).

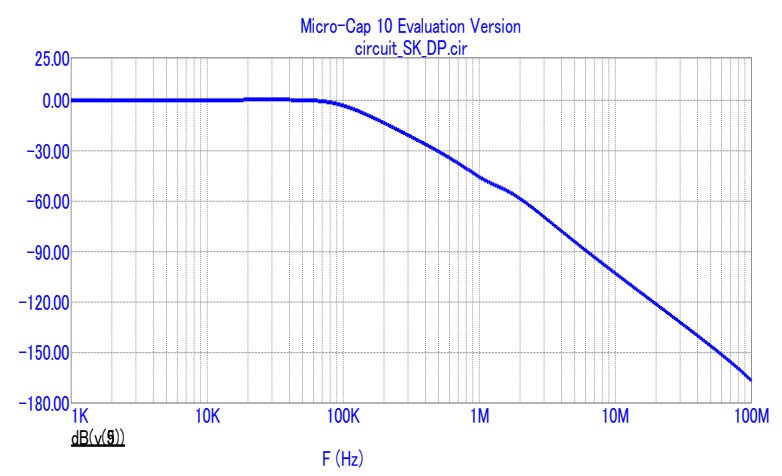

Fig. 7. Magnitude plot from simulation result of biquad with DISO in node 5 according to Fig.6.

Meanwhile, for the simple filter (output according to the node 4 ), the decreasing in the attenuation at high frequencies was still occurred (see Fig. 8).

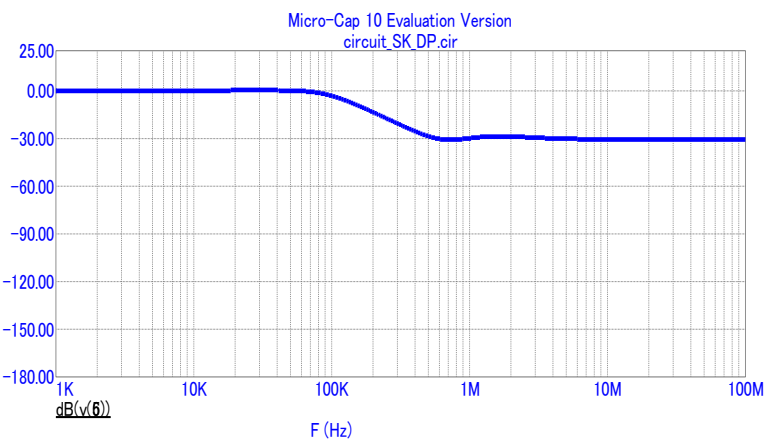

Fig. 8. Magnitude plot from simulation result biquad with DISO in node 4 according to Fig. 6.

The transfer function $\mathrm{T}$ of the circuit according to Fig. $3 b$ is solved by the graph in Fig. 4a. This graph is evaluated by Mason's formula (2).

$$
\begin{aligned}
& T(s)=\frac{V_{\text {OUT }}(s)}{V_{I N}(s)}=\frac{\sum p_{(i)} \cdot \Delta_{(i)}}{V-\sum S^{(K)} \cdot V^{(K)}}= \\
& =\frac{G \cdot G \cdot A}{(2 \cdot G+s 2 \cdot C) \cdot(G+s C)-G^{2}-s 2 \cdot C \cdot G \cdot A}
\end{aligned}
$$

where: $T$ is the transfer function, $V_{I N}, V_{O U T}$ are the input and output voltages, $G$ is the conductance of a working resistor in the filter, $A$ is the amplification factor of the operational amplifier, $C$ is the capacitance of the working capacitor in the filter.

The transfer function of the proposed circuit from Fig. 5 is solved by the graph in Fig. 4b. This graph is evaluated by Mason's formula (3).

We may see, that relations (2) and (3) are identical. This identity supplies the same magnitude plot at low and middle frequencies. But the circuit presented in Fig. 5 has a better response at high frequencies. Therefore, the transient frequency of used operational amplifiers has less influence on resulted magnitude plot.

$$
\begin{aligned}
& T(s)=\frac{V_{\text {OUT }}(s)}{V_{I N}(s)}=\frac{\sum p_{(i)} \cdot \Delta_{(i)}}{V-\sum S^{(K)} \cdot V^{(K)}}= \\
& =\frac{G \cdot G \cdot A}{(2 \cdot G+s 2 \cdot C) \cdot(G+s C)-G^{2}-s 2 \cdot C \cdot G \cdot A}
\end{aligned}
$$

\section{COMPARISON WITH OTHER SOLUTIONS}

Reference [4] described another circuit for the solving problem of decreasing of the attenuation of the low-pass filter at high frequencies.

The topology of this circuit is based on of the operational amplifier in the feedback branch with transfer $s 2 \cdot C$ (see Fig. 9).

The transfer function is constructed from the signal flow Mason-Coates (MC) graph of this circuit in Fig. 10 (4).

$$
\begin{aligned}
& T(s)=\frac{V_{\text {OUT }}(s)}{V_{I N}(s)}=\frac{\sum p_{(i)} \cdot \Delta_{(i)}}{V-\sum S^{(K)} \cdot V^{(K)}}= \\
& =\frac{G \cdot G \cdot A}{(2 \cdot G+s 2 \cdot C) \cdot(G+s C)-G^{2}-s 2 \cdot C \cdot G \cdot A^{2}}
\end{aligned}
$$

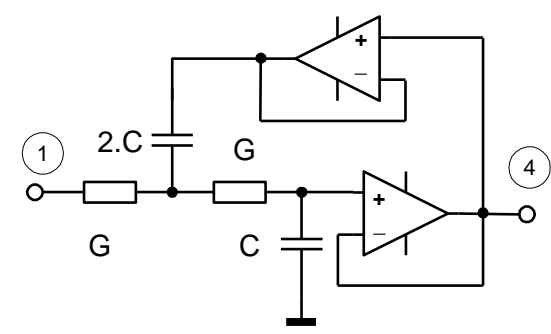

Fig. 9. Topology of thelow-pas filter with op-amp in the feedback branch.

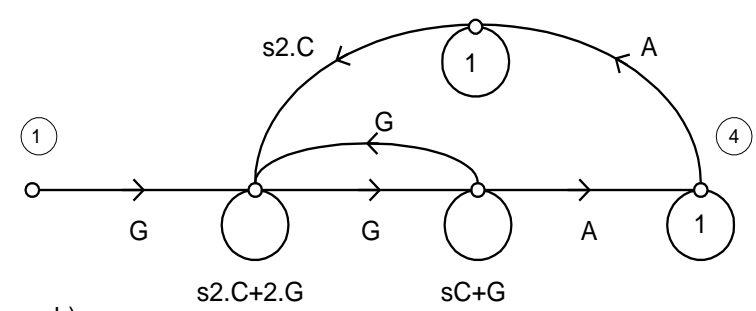

Fig. 10. Modified MC-graph corresponded to the circuit from Fig. 9.

As is shown, the formula (4) is different to equations (2) or (3), i.e. in the last member in the denominator has squared the factor A. However, if topology DISO of the operational amplifier is used as shown in Fig. 5, then the transfer function (3) is the same as the transfer function of the original biquad (2). 


\section{CONCLUSION}

The ineligible frequency response of the Sallen-Key low-pass filter of the even orders was explained and verified by computer simulation. The effect of a decreasing of the attenuation at high frequencies is caused by a decreasing the amplification factor $A$ in coincidence with finite series output resistance $g$ of the operational amplifier.

A decrease of the attenuation of the Sallen-Key low-pass filter structure of the even orders at high frequencies does not occur when the pair of operational amplifiers is used.

We explained that a pair of DISOs OPA in the cascade has not the same transfer function as the original biquad. But proposed circuit that is constructed from a pair of voltage followers in parallel form has the same transfer function as the original biquad. Moreover, the structure of this circuit can be derived from the MC-graph.

\section{REFERENCES}

[1] B. Carter, R. Mancini, Op Amps For Everyone, 5th ed. Oxford: Elsevier 2018 .

[2] J. Bicak, M. Leipert, M. Vlcek, A Linear Circuits an Systems, 1st ed. Prague: CTU publisher, 2007.

[3] T. Dostal, K. Vrba, The Electric filters, 1st ed. Brno: PC-DIR, 1997.

[4] S. Winder, Analogue and digital filter design. Elsevier, pp. 133-135, 2002.

[5] L. Thede, Practical analog and digital filter design. Artech House, Inc., pp. 87-95, 2004

[6] P. Martinek, P. Boreš, J. Hospodka, Electric filter. Prague: CTU Publisher, pp. 98-101, 2003.

[7] J. Puncochar, "Low Pass Filters Sallen and Key With Real Operational Amplifiers," Elektrorevue 10, 2005, pp. 1-13.

[8] T. Dostal, J. Mikula "Signal-Flow Graph Network Analysis". Radioengineering, 1992, vol. 1, no. 1,pp. 14-20.

[9] D. Biolek, Solving electronic circuits. Prague: BEN, 2004.

[10] K. Gorshkov, "Parameters extraction technique for optimal network functions of SC circuits," International Siberian Conference on Control and Communications (SIBCON), May 2015, pp. 215-220. 